Acta Cryst. (2002). A58 (Supplement), C206

ISOTOPIC MASS AND LATTICE CONSTANTS OF Si AND Ge: X-RAY STANDING WAVE MEASUREMENTS

J. Zegenhagen

European Synchrotron Radiation Facility, ESRF, Grenoble, France

Isotopic materials with tailored isotopic composition exhibit properties different from materials composed of the natural isotopic mixture according to their isotope abundance. They exhibit high thermal conductivities and their hardness can exceed the values of their natural counterparts. Electronic properties are influences as well via small changes in the electronic band structure and spin properties due to the isotope dependence of the nuclear spin. Even the lattice constant is a function of the isotopic mass, as recognized already in 1958 by London (1). The reason for this is the mass dependence of the zero-point motion of the atoms, i.e., purely quantum mechanical in origin. The variation of the lattice constant (or molar volume) with isotopic mass is small, in particular for elements with small relative mass difference of the isotopes and low Debye temperature, and almost vanishes at higher temperatures. For Si and especially for Ge the effect becomes too small to be measured with standard diffraction techniques, which furthermore would require perfect bulk crystals with the corresponding isotopic composition. We applied the X-ray standing wave technique to this problem (2) and determined the isotopic mass dependence of the lattice constant for $\mathrm{Si}$ and Ge from $30 \mathrm{~K}$ to room temperature with high accuracy (3).

References

(1) H. London, Z. Physik.Chem. 16, 302 (1958).

(2) A. Kazimirov, J. Zegenhagen, and M. Cardona, Science 282, 930 (1998).

(3) E. Sozontov, L.X. Cao, A. Kazimirov, V. Kohn, M. Konuma, M. Cardona, and J. Zegenhagen, Phys. Rev. Lett. 86, 5329 (2001).

Keywords: ISOTOPES, LATTICE CONSTANT, STANDING WAVES
Acta Cryst. (2002). A58 (Supplement), C206

\section{X-RAY STANDING-WAVE INVESTIGATIONS OF VALENCE} ELECTRONIC STRUCTURE

$\underline{\text { J. Woicik }}$

National Institute of Standards and Technology C/o Building 535-A

Brookhaven National Laboratory UPTON NY 11973 USA

We have examined the valence-electron emission from $\mathrm{Cu}, \mathrm{Ge}$, GaAs, InP, $\mathrm{NiO}$, and $\mathrm{TiO}_{2}$ single crystals under the condition of strong x-ray Bragg reflection; i.e., in the presence of the spatially modulated $x$-ray standing-wave interference field that is produced by the superposition of the incident and reflected x-ray beams. These crystals span the entire metallic, covalent, and ionic range of solid-state bonding. It is demonstrated that the valence-electron emission is closely coupled to the atomic cores, even for electron states close to a metallic Fermi edge. Using the bond-orbital approximation, the $\mathrm{x}$-ray standing-wave structure factor for valence-electron emission is derived in terms of the bond polarities and photoionization cross sections of the atoms within the crystalline unit cell and compared to experiment. Additionally, we demonstrate that by exploiting the spatial dependence of the electric-field intensity under Bragg condition, site specific valence electronic structure may be obtained. The technique is demonstrated for GaAs, $\mathrm{NiO}$, and $\mathrm{TiO}_{2}$ and compared to theory.

\section{Keywords: X-RAY STANDING-WAVE, PHOTOELECTRON SPECTROSCOPY}

\section{Acta Cryst. (2002). A58 (Supplement), C206 SOFT MODES AND FERROELECTRIC ORDER IN RELAXOR}

P. M. Gehring

National Institute of Standards and Technology NIST Center for Neutron Research 100 Bureau Drive, Stop 8562 GAITHERSBURG MD 20899-8562 USA

A review is given of our recent results from neutron scattering studies of the lead-oxide class of perovskite relaxors PMN and PZN. A soft ferroelectric TO mode has been identified in PMN at $1100 \mathrm{~K}$ that becomes overdamped at $\mathrm{Td}=$ $620 \mathrm{~K}$. This is the same temperature where polarized nanoregions (PNR) first begin to form, and thus suggests that a direct connection exists between the soft mode and the PNR. The concurrent appearance at Td of diffuse scattering intensity lends further support to this picture. at lower temperature the soft mode in PMN reappears close to $\mathrm{T}_{\mathrm{c}}=213 \mathrm{~K}$ (defined for $\mathrm{E}>\mathrm{Ec}$ ). These results are provocative in that the dynamics suggest a transition occurs from a relaxor state to an ordered ferroelectric state near $213 \mathrm{~K}$ in PMN, a system that exhibits no spontaneous polarization at any temperature. We discuss a coupled-mode model that has successfully described our data as well as those of earlier lattice dynamical studies of other perovskites such as $\mathrm{BaTiO}_{3}$.

\section{Keywords: RELAXOR FERROELECTRICS POLAR NANOREGIONS} LATTICE DYNAMICS

\section{Acta Cryst. (2002). A58 (Supplement), C206 \\ SHORT RANGE ORDERING AND POLARON DYNAMICS OF CMR MANGANITES}

D. Argyriou

Hahn-Meitner-Institut Sf-2 Glienicker Str 100 BERLIN 14109 GERMANY

Diffuse scattering measurements on a bilayer manganites that exhibit colossal magnetoresistance (CMR) exhibit a rich local structure arising from the shortrange ordering of Jahn-Teller active Mn(III). Quasielastic neutron scattering measurements show that these short-range polarons correlations are diffusive (non-propagating) and completely dynamic at high temperature, but then freeze upon cooling to a temperature $\mathrm{T} 310 \mathrm{~K}$. This glass transition suggests that the paramagnetic/insulating state arises from an inherent orbital frustration that inhibits the formation of a long range orbital- and charge-ordered state. Upon further cooling into the ferromagnetic-metallic state $\left(\mathrm{T}_{\mathrm{c}}=114 \mathrm{~K}\right)$, where the polarons melt, the diffuse scattering quickly develops into a propagating, transverse acoustic phonon.

Keywords: COLOSSAL MAGNETORESISTANCE, POLARON DYNAMICS, QUASIELASTIC NEUTRON SCATTERING 